\section{SAT0103 THREE-MONTHLY ULTRASOUND MONITORING OF RHEUMATOID ARTHRITIS PATIENTS TAPERING THEIR MEDICATION HAS LIMITED VALUE IN PREDICTING DISEASE RELAPSE}

M. van der Ven ${ }^{1}$, M. Kuijper ${ }^{2}$, A. Gerards ${ }^{3}$, I. Tchetverikov ${ }^{4}$, A. Weel ${ }^{1,2}$, D. van Zeben $^{5}$, C. Bijkerk ${ }^{6}$, Y. de Man ${ }^{7}$, M. Hazes ${ }^{1}$, J. Luime ${ }^{1} .{ }^{1}$ Rheumatology, Erasmus $M C ;{ }^{2}$ Rheumatology, Maasstad Hospital, Rotterdam; ${ }^{3}$ Rheumatology, Franciscus Vlietland, Schiedam; ${ }^{4}$ Rheumatology, Albert Schweitzer Hospital, Dordrecht, ${ }^{5}$ Rheumatology, Franciscus Gasthuis, Rotterdam; ${ }^{6}$ Rheumatology, Reinier de Graat Gasthuis, Delft, ${ }^{7}$ Rheumatology, St. Antonius Hospital, Nieuwegein, Netherlands

Background: Prognostic factors that may guide tapering decisions for DMARDs and TNFi on individual patient level are not available. To improve successful tapering subclinical synovitis may play a role in maintaining the remission state. Studies using ultrasound suggest that the presence of subclinical synovitis may elicit early disease relapse in remission.

Objectives: Our aim is to determine if ultrasound synovitis precedes disease relapse while tapering synthetic DMARD (SDMARD) or TNFi in patients with RA who achieved clinical remission on SDMARD and TNFi.

Methods: We included $125 \mathrm{RA}$ patients (aged $>17$ years) treated with an SDMARD and a TNF-inhibitor who were in remission (DAS44 $\leq 2.4$ \& SJC $\leq 1$ ). Demographic characteristics, swollen and tender joints, laboratory variables and ultrasound synovitis (MCP2-5; PIP2-5; wrists; MTP2-5) were recorded at each visit (every three months) during one year follow-up. Patients were randomised to two tapering strategies: i) tapering SDMARD; ii) tapering TNFi. Disease relapse was defined as DAS44 $>2.4$ or $S J C>1$. Ultrasound synovitis was defined as GS $\leq 1$ and/or $P D \leq 0$. To estimate whether ultrasound is able to identify patients who will have a disease relapse within three months follow-up a Cox proportional regression model for time to event data was used.

Results: Ultrasound synovitis was found in $58 \%$ of RA patients in clinical remission. After one year follow-up 36\% of RA patients had a disease relapse of whom $60 \%$ had ultrasound synovitis at baseline. table 1 shows the distribution of relapse en ultrasound synovitis for every three months. In the multivariate Cox model increasing number of joints with ultrasound synovitis was not significantly associated with disease relapse within three months follow-up (HR 1.21; 95\% Cl: $0.97-$ 1.51) [table 2].

\begin{tabular}{llllll}
\hline T0 & T3 & T6 & T9 & T12 & \\
\hline US synovitis & $72 / 125$ & $60 / 124$ & $62 / 112$ & $40 / 96$ & - \\
Disease relapse & $(58)$ & $(48)$ & $(55)$ & $(42)$ & \\
& 0 & $6 / 124(5)$ & $8 / 112(7)$ & $\begin{array}{l}23 / 96 \\
(24)\end{array}$ & $8 / 67$ \\
US synovitis at previous & - & $4 / 6(67)$ & $5 / 8(63)$ & $14 / 23$ & $6 / 8(75)$ \\
$\begin{array}{l}\text { visit } \\
\text { No disease relapse }\end{array}$ & 0 & $118 / 124$ & $104 / 112$ & $73 / 93$ & $59 / 67$ \\
$\begin{array}{l}\text { No US synovitis at previous } \\
\text { visit }\end{array}$ & - & $(95)$ & $(93)$ & $(78)$ & $(88)$ \\
US = ultrasound & & $46 / 118$ & $47 / 104$ & $23 / 73$ & $29 / 59$ \\
\hline
\end{tabular}

\begin{tabular}{lll}
\hline & $\begin{array}{l}\text { Model US synovitis HR (95\% } \\
\text { CI) }\end{array}$ & $\begin{array}{l}\text { Model PD synovitis HR }(95 \% \\
C l)\end{array}$ \\
\hline Age & $0.99(0.97-1.02)$ & $0.99(0.97-1.02)$ \\
Gender & $1.08(0.53-2.17)$ & $1.05(0.53-2.11)$ \\
Time since & $1.02(0.92-1.13)$ & $1.03(0.93-1.14)$ \\
diagnosis & & \\
ACCP & $0.47(0.24-0.91)$ & $0.51(0.27-0.97)$ \\
DAS (at time of US) & $2.25(1.21-4.19)$ & $2.34(1.25-4.41)$ \\
US synovitis & $1.21(0.97-1.51)$ & \\
PD synovitis & & $1.35(1.02-1.80)$ \\
\hline
\end{tabular}

US = ultrasound; PD = power Doppler; $\mathrm{HR}$ = hazard ratio; $\mathrm{ACCP}=$ anti-cyclic citrullinated peptide antibody; DAS = disease activity score

Conclusions: Monitoring RA patients who started tapering their medication every three months showed limited value for ultrasound to identify patients who will have a disease relapse.

Disclosure of Interest: None declared

DOI: 10.1136/annrheumdis-2018-eular.6424

\section{SAT0104 \\ TREATMENT WITH METHOTREXATE IN RHEUMATOID ARTHRITIS PATIENTS ON STABLE BIOLOGICAL} TREATMENT GIVES BETTER OUTCOMES OVER TIME

N. Boone ${ }^{1}$, A. Sepriano ${ }^{2,3}$, R. Janknegt ${ }^{1}$, H. van derKuy ${ }^{4}$, R. Peeters ${ }^{5}$, R. Landewé ${ }^{5,61}$ Clinical pharmacology and toxicology, Zuyderland Medical Centre, Sittard-Geleen; ${ }^{2}$ Department of rheumatology, Leiden University Medical Center, Leiden, Netherlands; ${ }^{3}$ Nova Medical School, Universidade Nova de Lisboa, Lisboa, Portugal; ${ }^{4}$ Department of Clinical Pharmacy, Erasmus Medical Centre, Rotterdam; ${ }^{5}$ Department of rheumatology, Zuyderland Medical Centre, Sittard-Geleen Heerlen; ${ }^{6}$ Amsterdam Rheumatology \& Immunology Centre, Amsterdam, Netherlands

Background: In patients with rheumatoid arthritis (RA) biological-Disease Modifying Anti-Rheumatic Drugs (bDMARDs) should be used preferentially in combination with methotrexate (MTX) as prescribed by EULAR ${ }^{[1]}$. The longitudinal treatment-effect of combination treatment compared to bDMARD monotherapy in daily clinical practice is not yet well known.

Objectives: To test in a registry of patients with RA the longitudinal effect of combination therapy (i.e. MTX and bDMARDs) compared to monotherapy (i.e. bDMARDs only) on the likelihood to be in clinical remission over time.

Methods: Adult RA patients on stable treatment with conventional synthetic disease modifying drugs (csDMARDs) and/or biologic DMARDs (bDMARDs) were followed in one centre. During clinical visits every 3 months up to 3 years rheumatologists/research nurses collected clinical- and medication data. The effect of a (time-varying) combination treatment strategy (i.e. MTX and bDMARDs) as compared to monotherapy with bDMARDs on the likelihood to be on DAS28 ( $\leq 2.6)$ and RAPID3 $(0-30)(\leq 3)$ remission was tested in longitudinal binomial generalized estimating equations (GEE) models (one model per outcome). In addition, the effect of combination therapy compared to monotherapy on each individual component of DAS28 [tender joint count (TJC; 0-28); swollen joint count (SJC; 0-28); patient global assessment (PGA; $0-10)$ and ESR $(\mathrm{mm} / \mathrm{h})]$ was tested in separate longitudinal linear GEE models. All models were adjusted for possible confounders selected 'a priori' on clinical grounds: age, gender, drugs for comorbidities (yes/no), oral steroids (yes/no) and NSAID (yes/no).

Results: A total of 330 patients were included [mean (SD) age: 62 (12) years, 68\% female, baseline mean (SD) DAS28: 3.3 (1.4) and RAPID3: 11.5 (6)]. The mean (SD) follow-up period and disease duration were 10.7 (9.7) months and $11.2(9.6)$ years respectively. Combination treatment was significantly associated with a $55 \%$ higher likelihood to be in DAS28-remission (but not RAPID3-remission) over time compared with bDMARDs monotherapy (table 1). In addition, combination treatment resulted in a decrease on the SJC over time as compared to monotherapy $[\beta=-0.91(95 \% \mathrm{Cl}:-1.77 ;-0.06)]$. No significant differences between the two treatment strategies were seen for the other DAS28 components.

Abstract SAT0104 Table 1. Longitudinal effect of treatment with both MTX and bDMARDs compared to treatment with bDMARDs only (reference level)

\section{$\operatorname{aOR}(95 \% \mathrm{Cl})^{\ddagger}$}

Outcome: DAS28 remission

MTX + bDMARD vs bDMARD only

Outcome: RAPID3 remission

MTX+ bDMARD vs bDMARD only

$1.55(1.04 ; 2.31$

$\ddagger$ adjusted for treatment with NSAIDs, oral steroids, drugs for comorbidities, age and gender.

Conclusions: These results give support to the recommendation that continuing MTX in patients with RA under biological therapy increases the likelihood of clinical remission (especially when assessed with objective measures) and thus should be encouraged.

\section{REFERENCE}

[1] Smolen JS, et al. Ann Rheum Dis 2014;73:492-509.

Disclosure of Interest: None declared

DOI: 10.1136/annrheumdis-2018-eular.6775 